\title{
Comb filters for the removal of transcranial current stimulation artifacts from single channel EEG recordings
}

\author{
Robert Guggenberger ${ }^{1, *}$ and Alireza Gharabaghi. ${ }^{1}$ \\ ${ }^{1}$ Institute for Neuromodulation and Neurotechnology, University Hospital Túbingen \\ cCorresponding author: robert.guggenberger@posteo.de
}

\begin{abstract}
The combination of transcranial alternating current stimulation (tACS) and electroencephalogram (EEG) for mobile and home-based interventions offers the potential for control and adaptation of stimulation parameters. Yet, during stimulation, the EEG is heavily affected by stimulation artifacts. Spatial filters are often unsuited because too few channels are recorded and hardware capabilities are limited. Due to their speed and as they can be used for single channels, we explore the performance of single-channel weighted comb filters on artifact removal. At any given time point $t$, the recording $r(t)$ is a superposition of a neurophysiological signal $n(t)$, the stimulation artifact a(t) and noise e(t). Now, we can estimate the artifact $\mathrm{a}(\mathrm{t})$ based on the recording from an earlier (or later) time-point shifted by the artifacts period. A weighted estimate based on multiple time points has the potential to improve the signal recovery. Therefore, we explored several approaches and evaluated their performance on simulated and real data. The comb kernel filters were implemented in Matlab (https://github.com/agricolab/ARtACS) and Python (https://github.com/agricolab/pyARtACS), and the code is open access under an X11-license. We found that independent of the weighting function, all comb filters exhibit similarity in their suppression of the DC component, the artifacts frequency, and its harmonics. Yet, different weighting functions exhibit different pass-band performance, evident as ringing and amplification, and their induction of time-domain echoes. Interestingly, we note that a causal uniform filter is comparable to more complex approaches, while offering the option for real-time filtering. Comb filters are able to remove tACS artifacts even if only a single channel is available. As comb filters require no assumptions about the shape of the artifact, they might also be useful for filtering of non-sinusoidal, e.g. pulsed or saw-tooth, transcranial current stimulation.
\end{abstract}

\section{Introduction}

https://doi.org/10.1515/cdbme-2021-2097

The combination of transcranial alternating current stimulation (tACS) and electroencephalogram (EEG) has been explored in several recent studies. While the analysis of EEG before or after stimulation posits limited technical challenges, the EEG recording during stimulation is heavily affected by the stimulation artifact. This has been linked to the spatial distribution, the nonstationary amplitude and the distorted sinusoidality of the artifact. It has been argued that unregularized spatial filters might be able to remove the stimulation artifact (Neuling et al., 2017). But if only few channels are recorded, the estimation of the spatial covariance can be insufficient. In the single-channel-case, spatial filters are impossible. Additionally, it has been reported that the spatial covariance is itself not stationary and that the artifact encompasses a large spatial subspace (Noury et al., 2016). Consider the assumption that the artifact is stationary and superpositioned on the physiological signal. Then, modulations in the amplitude of the recorded EEGsignal must be caused by changes in the underlying physiology. This would be the case, even if frequency and phase are matched to the stimulation signal. Approaches assuming such stationarity of the stimulation artifact have been used e.g. by Pogosyan et al. (2009). Yet, practical experience suggests that the stimulation artifact is actually not stationary. As a recording can exhibit an amplitude modulation of the stimulation artifact when the clocks of stimulation and recording device are not synchronized, this has been suggested as a cause for amplitude modulations. It has also been suggested that the closed-loop current control process within the stimulator might be a further source of amplitude modulation (Neuling et al., 2017). The temporally and spatially uneven impedance distribution has been suggested as cause of distortion. Non-linearites in the amplifier slew rate can distort the shape when the signal is close to the saturation threshold. Additionally, the amplitude has been reported to be modulated by heart-beat and respiration (Noury et al., 2016). Event-related modulation of skin impedance can also affect the scalp conductance at stimulation electrodes. In conclusion, recovering the true signal from the artifacted recording is technically challenging primarily because of such non-stationarities in the artifacts amplitude.

Methods based on adaptive template construction and temporal principal component analysis (tPCA) (Niazy et al. 2005) have been explored for removal of non-stationary and misshaped tACS artifacts (Helfrich et al., 2014). Consider that the process of template construction, the estimation of accurate weights for removal by template subtraction and the subsequent removal of residual artifacts using tPCA is computationally cumbersome. Additionally, it often requires offline analysis supported by visual inspection. Such a multistaged template-approach is therefore of limited utility for on-line artifact removal, especially in a single-channel case.

It should be noted that simulation studies suggested that the power of endogenous oscillations increases if the frequency of tACS matches the targets eigenfrequency (Kutchko and $\mathrm{Fr}$ hlich, 2013; Zaehle et al., 2010). This has been supported by evidence from animal studies (Schmidt et al., 2014), and human studies combining tACS with transcranial magnetic stimulation (TMS) (Guerra et al., 2016), or contrasting pre and post resting state power analysis (Zaehle et al., 2010). It has also been suggested that the phase of neuronal populations would be locked to the phase of the tACS signal (Reato et al., 2013). This has been supported by evidence from studies combining tACS with motor output (Brittain et al., 2013), TMS (Raco et al., 2016; Nakazono et al., 2016) or sensory perception (Gundlach et al., 2016). At the same time, eventlocked modulation of skin impedance can mimic phase and/or frequency locked modulations. Events might even be endogenous, e.g. heart beats (Noury et al., 2016). This suggests that transient and event-locked modulations in frequency and phase matching to the tACS can make the distinction between artifact and neurophysiological effect impossible when we accept non-sinusoidal distortion.

We were interested in development of a computationally fast approach, feasible for online artifact removal in a single channel, and usable in embedded systems with limited computational power. The approach was required to account for the dynamical modulation of the artifact amplitude, and the possibility of non-sinusoidal distortion.

\section{Approach}

The main idea is that at any given time point $t$, the recorded signal $r(t)$ is a linear superposition of a neurophysiological signal $n(t)$, the stimulation artifact $a(t)$ and a white noise term $e(t)$. The task is to recover $n(t)$ by estimating $a(t)$ as $\hat{a}(t)$ and subtracting it from $r(t)$. Assume that the tACS artifact were non-sinusoidal, but stationary and periodic. At the same time, assume that neurophysiological signals $n$ and noise $\epsilon$ were absent. Then, we could estimate the amplitude of $a$ at any 
time-point $t$ by using the signal $r$ recorded from any time-point, as long as this time-point is an integer multiple of the artifacts period length $p$ earlier (1). Subtraction of a delayed version of the signal is also known as comb filter. Please note that for discretely sampled signals, this approach natively supports only frequencies which are integer divisibles of the sampling frequency. If this is not the case, upsampling the recorded signals to an appropriate sampling rate, comb filtering, and downsampling again is a viable approach.

$$
\hat{a}(t)=r(t-n p)
$$

\section{$n \in \mathbb{Z}$}

\subsection{Uniform Comb Filter}

Consider that if noise term $\epsilon$ is were white, and because the expectation of white noise $\langle\epsilon\rangle$ converges asymptotically to zero with increased sample size, an approach to estimate a bias-free artifact amplitude would be to average across as many earlier periods as possible (2). Subsequently, this estimate can be used to remove the artifact from $r$. In real applications, stimulation duration is limited and computational constraints exist. This is reflected by the fact that we have to use a finite number for $N$.

$$
\hat{a}(t)=\sum_{n=1}^{N} \frac{r(t-n p)}{N}
$$

Please note that averaging across neighboring periods $M(3)$ has been suggested before and termed superposition of moving averages (SMA) by Kohli and Casson (2015).

$\hat{a}(t)=\sum_{n-M / 2}^{n+M / 2} \frac{r(t-n p)}{M+1}$

Consider that the approach using only past values (2) returns a causal filter. Applied online, a causal filter would be able to remove the artifact without the delay of $(M p) / 2$ necessary for SMA. Furthermore, SMA is well-defined only for even M. This motivates the exploration of causal filters for artifact removal.

\subsection{Temporal Weighting}

Consider that the amplitude of the artifact has been described to be non-stationary and dynamically modulated (Noury et al., 2016; Neuling et al., 2017). Although it has been suggested that there are event-dependent components of the amplitude modulation (e.g. by heartbeat or respiration Noury et al. (2016) or stimulator impedance check (Neuling et al. (2017)), the parameters of the dynamical system governing eventindependent amplitude modulation are usually not known a priori. This can render online artifact removal problematic. One approach to tackle this problem is to use instead of a constant weight $1 / N(4)$, a lag-dependent weighting function $w_{n}(4)$.

$\hat{a}(t)=\sum_{n=1}^{N} w_{n} r(t-n p)$

By controlling the parameters of the weight functions, we can attempt to match the process governing the amplitude modulation. This might allow us to achieve a better artifact estimation and subsequent removal. Please note that if the reliability of past periods can be estimated, we might select weights empirically, e.g. with autocorrelation. Yet, these approaches require extended calculation periods and/or offline analysis. There are a variety of options for designing the filter weights, from linear decreasing weighting, exponential weighting or Gaussian weighting. Besides these real-valued kernels one could use complex-valued kernels which would natively support non-integer frequencies at the cost of assuming sinusoidality or estimate the weight at each period from the auto-correlation across periods.

\subsubsection{Justification by Sampling}

Consider for example the simple one-step comb filter, where we remove the artifact by subtracting a value sampled from any earlier period. Assume now that the sample would be drawn at random from the last $N$ periods with uniform distribution. If the system governing amplitude modulation were non-stationary, we would expect that the accuracy of the estimate degrades the longer the delay, while the precision improves by increasing the number of periods on which we base our estimate. This rationale justifies nonuniform weights, and to use weights that are designed to increase precision and accuracy of the estimate.

\subsubsection{Justification by AR (1) process}

Consider that the system governing the amplitude of the stimulation artifact could be decomposed into the constant amplitude $c$ controlled by the stimulator and a dynamical, event-unrelated process governing the amplitude modulation. If we model this process as an AR (1) process, and the kernel behind the modulation of the artifacts amplitude were known, or could be estimated sufficiently, we could construct an optimal weighting function as a deconvolution filter. Note that, for practical applications, we would need kernels decaying sufficiently fast to be computationally feasible.

\subsection{Implementation and Evaluation}

We implemented kernel creation and artifact removal in Matlab (https://github.com/agricolab/ARtACS) and Python (https://github.com/agricolab/pyARtACS), and the code is open access under an X11-license. The set of possible kernels is infinite, so we selected several kernel approaches based on mathematical interest, and evaluated their performance on simulated and real data against two alternative approaches, the removal of a sinusoid, or of periodic principal components. We selected these alternative approaches, to contrast with an approach limited to sinusoids, and one that is usually run offline and computationally demanding but could theoretically adapt its template better to the data at hand.

We explored different filters for $10 \mathrm{~Hz}$, all with a period number of 10 . The causal DFT filter was applied recursively not only at 10 , but also at 20,30 , and $40 \mathrm{~Hz}$ to reduce harmonics. Based on the filtered recordings with stimulation and the average response in the stimulation-free recording, we calculated the $R^{2}$ value. Blue bars indicate the average $R^{2}$ for single-trial reconstructions, and red bars the average $\mathrm{R}^{2}$ for boot-strapped grand-average ERPs based on 100 epochs (see figure 1, 2 and table 1).

The simulated signal was created as superposition of a $10 \mathrm{~Hz}$ tACS-artifact, and an event-related potential (ERP). The ERP was simulated as the gradient of a flat top window. The amplitude of the artifact was simulated as a distorted, nonstationary sinusoidal. The initial stimulation signal was modeled as sinusoidal signal. We distorted it by adding periodic white noise. Additionally, the stimulation amplitude was driven by an Ornstein-Uhlenbeck process with a stiffness 0.5 , and a Hanning window time-locked to the event-related potential. In that way, we were able to simulate event- 
independent modulations of the artifacts amplitude, and modulations locked to an event. We simulated 100 trials with a length of 8000 samples (see figure 1).

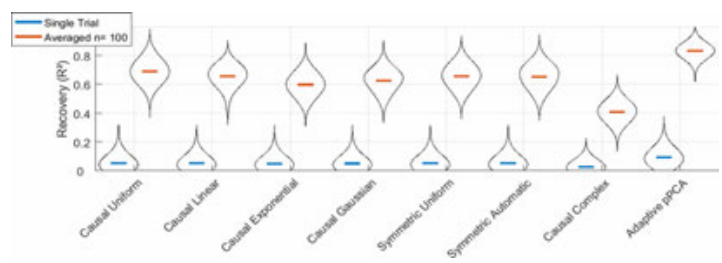

Figure 1: Overview for Artifact Removal from Simulated Signal. with kernel density estimates of the $R^{2}$ recovery values for different approaches

Consider that tACS can have physiological effects on cortical ERP. To properly evaluate the recovery using the filtering approaches, we needed a physiological signal unlikely to be affected by tACS.

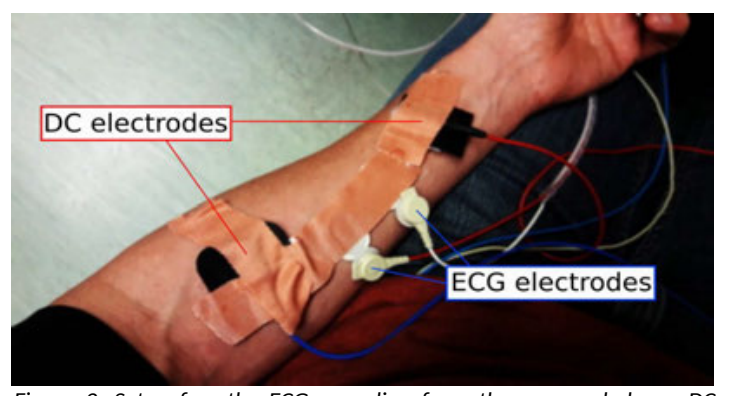

Figure 2: Setup for the ECG recording from the arm and how $D C$ electrodes were applied. An additional, artifact-free ECG was recorded with bipolar electrodes attached at the chest.

We therefore measured electrocardiogram (ECG) at the flexor digitorum of the left upper limb, while we stimulated distal and proximal with tACS to the recording electrodes (see figure 2). We also recorded ECG at the chest, and detected the R-peak for epoching the data. Please note that no tACSartifact was visible in the chest ECG. Physiological signals were acquired (BrainAmp DC, BrainProducts, Munich, Germany) at a sampling rate of $1 \mathrm{kHz}$ and low-pass filtered below $35 \mathrm{~Hz}$. Stimulation was delivered (NeuroConn DC Stimulator Plus, neuroConn $\mathrm{GmbH}$, Ilmenau, Germany) with carbon rubber electrodes and electrode gel (Ten20, Weaver and Company, Aurora, USA) with an intensity of $1 \mathrm{~mA}$ at a frequency of $11 \mathrm{~Hz} .1 \mathrm{~mA}$ is a typical intensity used in most tDCS studies, and $11 \mathrm{~Hz}$ was used as it results in non-integer period lengths at the typical EEG sampling rate of $1 \mathrm{kHz}$, challenging the comb filter approach maximally. We detected the R-peak based on the chest-recordings, which allowed us to cut epochs of $8 \mathrm{~s}$ duration for the recording at the upper limb, for a total of 134 R-peaks without stimulation and 136 with stimulation. We estimated the true shape of the R-peak by taking the average of the stimulation-free signal (see figure 3).

\section{Discussion}

We found that all approaches were able to recover the signal, especially for averaged signals (see table 1). It should be noted that the weighted filters exhibited slightly average lower $\mathrm{R}^{2}$ values and a slightly more skewed distribution. This might be linked to distortion by echoes or insufficient artifact removal in a few trials. The causal complex filter performed worst, although we filtered not only the main frequency of the artifact, but also the harmonical frequencies. This is most likely caused by the non-sinusoidality of the artifact. Most interestingly, the causal uniform filter performed on par with the symmetric filters, regardless of whether these were weighted uniform or based on the autocorrelation. This suggests that the causal uniform filter can be highly effective, in spite of its low computational complexity,

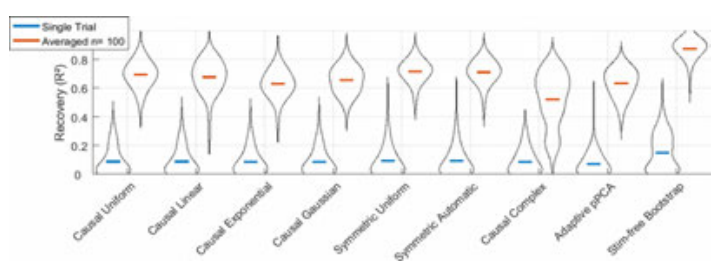

Figure 3: Overview for Artifact Removal from Upper Limb Electrocardiogram with kernel density estimates of the $R^{2}$ recovery values for different approaches, including the stim-free bootstrap as the true signal is unknown in the empirical case.

\begin{tabular}{c|cc|cc} 
& \multicolumn{2}{|c|}{ Simulation } & \multicolumn{2}{c}{ ECG } \\
& $\begin{array}{c}\text { Single } \\
\text { Trial }\end{array}$ & $\begin{array}{c}\text { Grand } \\
\text { Average }\end{array}$ & $\begin{array}{c}\text { Single } \\
\text { Trial }\end{array}$ & $\begin{array}{c}\text { Grand } \\
\text { Average }\end{array}$ \\
\hline Causal Uniform & 0.05 & 0.689 & 0.089 & 0.694 \\
Causal Linear & 0.049 & 0.657 & 0.089 & 0.677 \\
Causal Exponential & 0.048 & 0.599 & 0.085 & 0.628 \\
Causal Gaussian & 0.048 & 0.626 & 0.084 & 0.655 \\
Symmetric Uniform & 0.052 & 0.659 & 0.089 & 0.717 \\
Symmetric Automatic & 0.053 & 0.656 & 0.092 & 0.707 \\
Causal Complex & 0.028 & 0.409 & 0.08 & 0.517 \\
Adaptive PCA & 0.093 & 0.838 & 0.065 & 0.629 \\
Stim-Free Bootstrap & n.a. & n.a. & 0.142 & 0.87
\end{tabular}

Table 1: Numerical results of the average $R^{2}$ value for the reconstruction of the signal for simulated and electrocardiogram data

3.1 Echos

A key disadvantage of comb filters is that while they subtract the artifacts, any underlying physiological signal will cause a periodic echo according to the weights of the kernel (see figure 4). This means that strong weights close to the kernels center might increase the precision of the artifact amplitude estimate, but can also induce unwanted and strong echos close to the occurrence of a physiologically interesting event. Additionally, if the duration of the physiological signal is longer than the period of the kernel, the signal will be distorted by the echo. This could be addressed by delaying the kernel, i.e. kernels with zero weights for a number of periods before their estimation weights start, or inverting the kernels, i.e. kernels with increasing instead of decreasing weights with increasing delay, or applying causal filters piece-wise left and right of the signal.
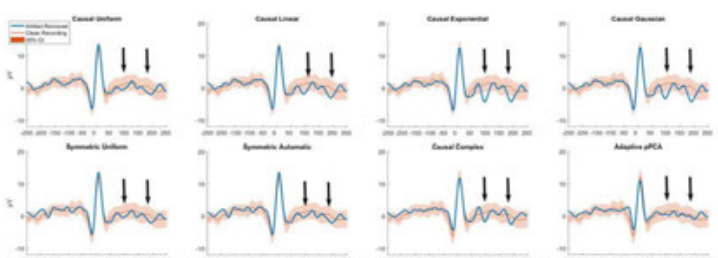

Figure 4: Overview of Reconstruction in the time domain highlights possibility of echos. Black arrows highlight where echoes are expected and also clearly visible for some kernels. 


\section{Conclusion}

We note that all filters were able to recover the signal, and that the causal uniform filter is comparable to computationally more complex approaches. This suggests its utility for environments with limited computational power, or for parallel application on multiple channels. Furthermore, being a purely causal filter, it can achieve online performance at high speed and with minimal delay. Finally, note that comb filters are purely periodic with no assumptions about the shape of the artifact. Comb filters might therefore be useful for filtering of deliberately non-sinusoidal, e.g. pulsed (Jaberzadeh et al., 2015) or misshaped (Cole and Voytek, 2017) transcranial current stimulation.

\section{References}

Brittain, J.-S., Probert-Smith, P., Aziz, T. Z., and Brown, P. (2013). Tremor suppression by rhythmic transcranial current stimulation. Current Biology, 23(5):436 440, DOI: 10.1016/j.cub.2013.01.068.

Cole, S. R. and Voytek, B. (2017). Brain oscillations and the importance of waveform shape. Trends in Cognitive Sciences, 21(2):137 149, DOI: 10.1016/j.tics.2016.12.008.

Guerra, A., Pogosyan, A., Nowak, M., Tan, H., Ferreri, F., Lazzaro, V. D., and Brown, P. (2016). Phase dependency of the human primary motor cortex and cholinergic inhibition cancelation during beta tACS. Cerebral Cortex, 26(10):3977 3990, DOI: 10.1093/cercor/bhw245.

Gundlach, C., M ller, M. M., Nierhaus, T., Villringer, A., and Sehm, B. (2016). Phasic modulation of human somatosensory perception by transcranially applied oscillating currents. Brain Stimulation, 9(5):712 719, DOI: 10.1016/j.brs.2016.04.014.

Helfrich, R. F., Schneider, T. R., Rach, S., Trautmann-Lengsfeld, S. A., Engel, A. K., and Herrmann, C. S. (2014). Entrainment of brain oscillations by transcranial alternating current stimulation. Current Biology, 24(3):333 339, DOI: 10.1016/j.cub.2013.12.041.

Jaberzadeh, S., Bastani, A., Zoghi, M., Morgan, P., and Fitzgerald, P. B. (2015). Anodal transcranial pulsed current stimulation: The e ects of pulse duration on corticospinal excitability. PLOS ONE, 10(7):e0131779, DOI: 10.1371/journal.pone.0131779.

Kohli, S. and Casson, A. J. (2015). Removal of transcranial a.c. current stimulation artifact from simultaneous EEG recordings by superposition of moving averages. In 2015 37th Annual International Conference of the IEEE
Engineering in Medicine and Biology Society (EMBC). IEEE, DOI: 10.1109/embc.2015.7319131.

Kutchko, K. M. and Fr hlich, F. (2013). Emergence of metastable state dynamics in interconnected cortical networks with propagation delays. PLoS Computational Biology, 9(10):e1003304, DOI: 10.1371/journal.pcbi.1003304.

Nakazono, H., Ogata, K., Kuroda, T., and Tobimatsu, S. (2016). Phase and frequency-dependent e ects of transcranial alternating current stimulation on motor cortical excitability. PLOS ONE, 11(9):e0162521, DOI: 10.1371/journal.pone.0162521.

Neuling, T., Ruhnau, P., Weisz, N., Herrmann, C. S., and Demarchi, G. (2017). Faith and oscillations recovered: On analyzing EEG/MEG signals during tACS. Neurolmage, 147:960 963, DOI: 10.1016/j.neuroimage.2016.11.022.

Niazy, R., Beckmann, C., Iannetti, G., Brady, J., and Smith, S. (2005). Removal of FMRI environment artifacts from EEG data using optimal basis sets. Neurolmage, 28(3):720 737, DOI: 10.1016/j.neuroimage.2005.06.067.

Noury, N., Hipp, J. F., and Siegel, M. (2016). Physiological processes non-linearly a ect electrophysiological recordings during transcranial electric stimulation. Neurolmage, 140:99 109, DOI:

10.1016/j.neuroimage.2016.03.065.

Pogosyan, A., Gaynor, L. D., Eusebio, A., and Brown, P. (2009). Boosting cortical activity at beta-band frequencies slows movement in humans. Current Biology, 19(19):1637 1641, DOI: 10.1016/j.cub.2009.07.074.

Raco, V., Bauer, R., Tharsan, S., and Gharabaghi, A. (2016). Combining TMS and tACS for closedloop phase-dependent modulation of corticospinal excitability: A feasibility study. Frontiers in Cellular Neuroscience, 10, DOI: 10.3389/fncel.2016.00143.

Reato, D., Rahman, A., Bikson, M., and Parra, L. C. (2013). E ects of weak transcranial alternating current stimulation on brain activity a review of known mechanisms from animal studies. Frontiers in Human Neuroscience, 7, DOI: 10.3389/fnhum.2013.00687.

Schmidt, S. L., Iyengar, A. K., Foulser, A. A., Boyle, M. R., and Fr hlich, F. (2014). Endogenous cortical oscillations constrain neuromodulation by weak electric elds. Brain Stimulation, 7(6):878 889, DOI: 10.1016/j.brs.2014.07.033.

Zaehle, T., Rach, S., and Herrmann, C. S. (2010). Transcranial alternating current stimulation enhances individual alpha activity in human EEG. PLoS ONE, 5(11):e13766, DOI: 10.1371/journal.pone.0013766. 